\title{
La politique des expositions temporaires
}

Hélène Fulgence, Philippe Descola, Lilian Thuram et Roger Boulay

\section{(2) OpenEdition}

\section{Journals}

Édition électronique

URL : https://journals.openedition.org/actesbranly/753

DOI : $10.4000 /$ actesbranly.753

ISSN : 2105-2735

Éditeur

Musée du quai Branly Jacques Chirac

\section{Référence électronique}

Hélène Fulgence, Philippe Descola, Lilian Thuram et Roger Boulay, « La politique des expositions temporaires », Les actes de colloques du musée du quai Branly Jacques Chirac [En ligne], 8 | 2017, mis en ligne le 12 juin 2017, consulté le 21 septembre 2021. URL : http://journals.openedition.org/ actesbranly/753; DOI : https://doi.org/10.4000/actesbranly.753

Ce document a été généré automatiquement le 21 septembre 2021.

(C) Tous droits réservés 


\section{La politique des expositions temporaires}

\section{Hélène Fulgence, Philippe Descola, Lilian Thuram et Roger Boulay}

Hélène Fulgence, Philippe Descola, Lilian Thuram et Roger Boulay

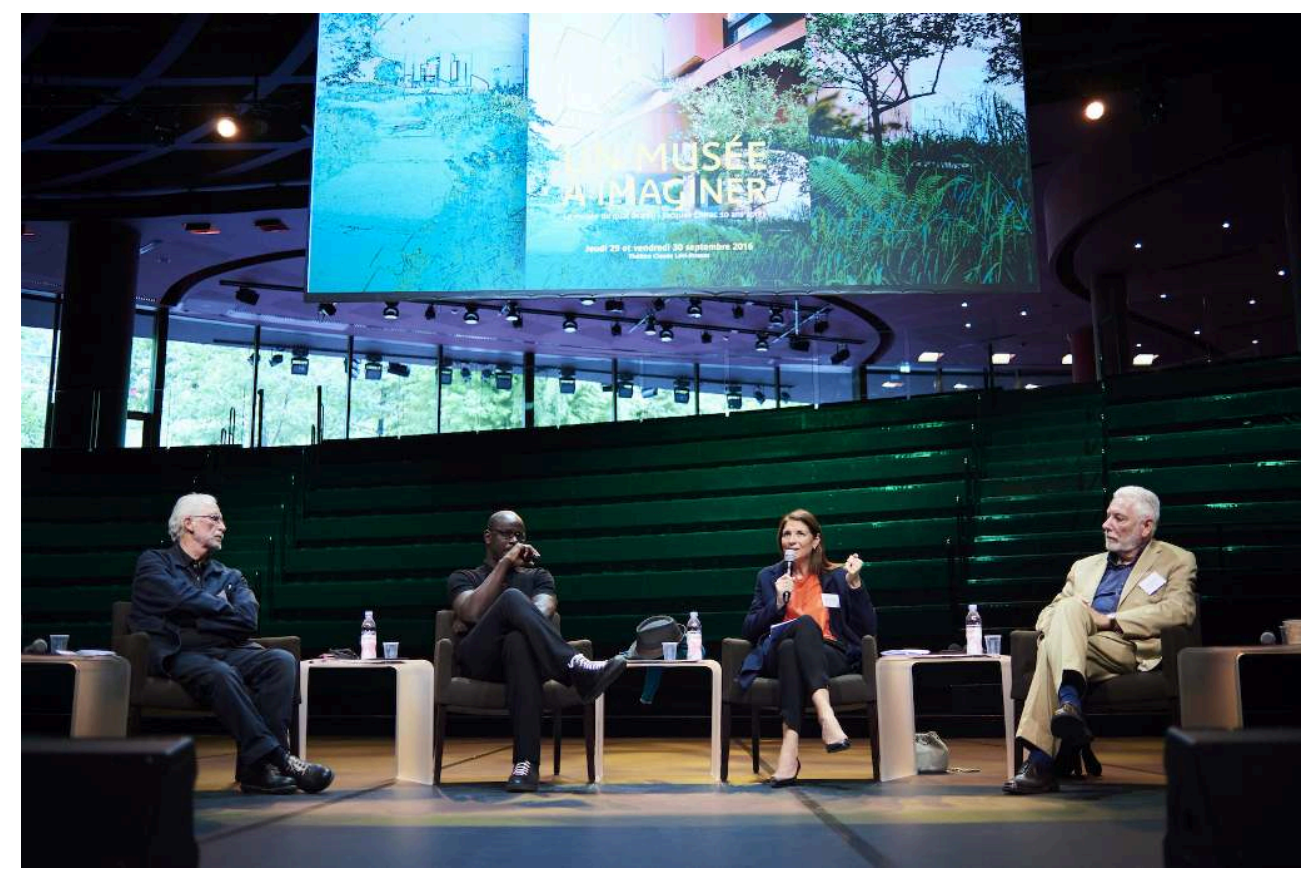

(C) musée du quai Branly - Jacques Chirac, photo Cyril Zannettacci

\section{Mme Hélène FULGENCE}

Le thème qui m'a été proposé pour animer cette table ronde porte sur la question des commissariats extérieurs que nous avons sollicités pour les expositions du musée du quai Branly - Jacques Chirac. Compte tenu des commissaires avec lesquels j'ai travaillé, j’ai envie de réfléchir sur ce qu'est exposer de la pensée ou de l'ethnographie. Cela a été 
un enjeu très important pour le musée du quai Branly - Jacques Chirac. Nos discussions vont tourner autour de ce sujet.

2 Tout à l'heure, nous avons beaucoup parlé de la manière d'exposer les collections permanentes. En réalité, le musée du quai Branly - Jacques Chirac a été conçu dans un va-et-vient très important entre le discours proposé par les expositions temporaires et celui proposé par les collections permanentes. Depuis l'ouverture, il y a eu 97 expositions temporaires. Elles sont le lieu de la pluralité des points de vue, de l'éclectisme, de la vision des auteurs. À la limite, le musée aurait pu se contredire sur ces 97 expositions et, sur un même thème, avoir présenté deux thèses diamétralement opposées. Cette programmation a aussi permis de relier la collection à des points de vue décalés, à des points de vue contemporains et à une réflexion non seulement historique, mais sur le monde tel qu'il est aujourd'hui. C'est un élément important, que le public du musée a compris très tôt. Pour évoquer la réception de ces expositions, dès la troisième ou quatrième année, le public francilien, qui constitue la majorité du public du musée, avait tendance à venir 2,5 fois par année pour voir des expositions. C'est bien le signe que le public a très bien compris qu'il y avait une pluralité de discours et que l'on pouvait se nourrir de manière très différente.

3 Laissons chacun des intervenants se présenter rapidement et dire de quelles expositions il a été commissaire au musée.

\section{Philippe DESCOLA}

4 Je suis anthropologue. J'enseigne au Collège de France et à l'école des Hautes Études en sciences sociales. J'ai eu le grand plaisir de faire une exposition il y a maintenant 7 ans qui s'appelait La fabrique des images et qui était une exposition dite anthropologique. Elle avait pour objectif de développer un point de vue anthropologique sur les images.

\section{Lilian THURAM}

Je suis un ancien joueur de football. Je suis Président de la fondation Éducation contre le racisme. L'idée est que l'on puisse se questionner et que l'on questionne la société pour comprendre que chacun de nous est le fruit d'un passé qui s'est construit sur des hiérarchies liées aux couleurs de peau, à la sexualité ou au genre. Il s'agit, notamment chez les enfants, d'essayer de développer des questionnements pour ne pas reproduire ces hiérarchies et pour essayer de construire une société plus égalitaire. En 2011, l'exposition Exhibitions, l'invention du sauvage avait essayé de montrer aux visiteurs qu'il n'y a pas si longtemps que cela l'on pouvait voir des hommes, des femmes ou des enfants dans des enclos, des zoos, des cirques, des foires et que cela a construit un regard, des catégories, ainsi que des hiérarchies.

\section{Roger BOULAY}

6 Je suis un ancien du musée de la Porte Dorée. Après avoir fait, avec le centre Tjibaou à Nouméa et le musée de la Porte Dorée, une exposition qui s'appelait Cannibales et Vahinés, il y a 16 ans, j'ai eu l'opportunité et le plaisir, grâce à Stéphane Martin, Hélène Fulgence et Yves Le Fur, de continuer dans cette veine en faisant ici Festetics de Tolna, un voyageur hongrois dans le Pacifique des années 1893. Ensuite Tarzan. Dans un autre domaine, mais quand même avec un grand souci de travailler sur le regard des uns et des autres, j'ai pu faire avec Emmanuel Kasarhérou l'exposition Kanak. Je pense être en préparation d'une future proposition sur l'imagerie, les stéréotypes qui s'appellera Le magasin des petits explorateurs.

\section{Mme Hélène FULGENCE}


7 Je propose d'aborder le sujet en deux temps. La première question, qui s'est posée à nous tous, à la fois au comité de programmation et à la Directrice de production que je suis, est : comment exposer de la pensée avec le média exposition qui a sa pauvreté et qui peut être rigide? Quelle qualité de réponse le média exposition a-t-il apporté aux expositions de nos commissaires?

8 Philippe, vous avez publié Nature et culture en 2005. À partir de là, nous vous sollicitons quelques années plus tard pour vous demander si ce livre pourrait fournir la matière d'une exposition. Vous répondez oui. Ce serait bien que vous rappeliez le postulat de ce livre.

\section{Philippe DESCOLA}

Commençons même plus en amont et parlons des expositions temporaires. Initialement, le musée a été conçu avec ce double dispositif: les collections permanentes et les expositions temporaires. C'est un mouvement de fond qui a saisi les musées d'ethnographie depuis une trentaine d'années. L'une des figures centrales de ce mouvement est Jacques Hainard au musée de Neufchâtel qui avait développé l'idée que, de façon à mettre un peu de distance critique vis-à-vis des objets exposés dans les collections permanentes, il était indispensable d'avoir des expositions temporaires qui servent de contrepoint, quelquefois d'ailleurs de critique même du dispositif général de l'exposition permanente qui est un dispositif qui est relativement récent dans l'histoire de la muséographie puisqu'il date du début du XXe siècle. Au XIX ${ }^{\mathrm{e}}$, nous avions de très nombreuses autres formes de muséographies ethnographiques, dont il ne subsiste plus de traces en dehors du musée Pitt Rivers à Oxford qui est fondé sur la généalogie des formes. Il y avait des musées diffusionnistes, des musées évolutionnistes. La disposition par le fait qu'un objet prend son sens parce qu'il est relié à d'autres objets produits à l'intérieur d'une même culture et que cette culture a un sens parce qu'elle est reliée à d'autres cultures à l'intérieur d'une même aire et que cette aire à un sens parce qu'elle est située dans une aire géographique, c'est une idée récente. C'est une idée qui a été inventée par Philipp Franz von Siebold au XIX ${ }^{e}$ siècle et qui a mis beaucoup de temps à faire son chemin, mais qui maintenant domine dans tous les musées d'ethnographie. D'où la nécessité des expositions temporaires qui viennent effriter, contourner, critiquer ce dispositif général et apporter des informations supplémentaires que les collections permanentes ne permettent pas d'apporter.

10 Au fil des années, j'avais développé une théorie anthropologique fondée sur l'idée qu'il y a un nombre fini de façons de percevoir les discontinuités entre humains et non humains. Ces dernières sont fondées sur l'idée que n'importe quel élément dans mon environnement peut soit manifester une intention, une intériorité, quelque chose que je me reconnais à moi-même aussi comme humain, ou bien au contraire en être complètement dépourvu et que je peux reconnaître dans cet élément de mon environnement des dispositions physiques qui sont analogues aux miennes ou qui sont différentes. Le résultat de tout cela est un système que j'ai appelé des ontologies, c'està-dire des manières de percevoir des éléments saillants dans l'environnement qui sont au nombre de quatre. J'avais essentiellement développé cette théorie à partir de textes (monographies ethnographiques, travaux d'historiens, travaux de philosophie, de philologie, de théologie). Bien sûr, ces documents reflétaient assez bien l'esprit de tel ou tel peuple, culture ou civilisation. Une fois ce travail accompli, je me suis demandé s'il y avait un moyen de vérifier cela dans un autre domaine que le domaine discursif ou du discours en général rapporté par des textes dans les images. J'ai donc commencé à 
travailler sur les images pour voir si l'on pouvait repérer dans les images le genre de distinctions que j'avais repérées dans des textes. C'était expérimental. C'est là que la question d'une exposition s'est posée, qui était d'une certaine façon une expérience dans l'expérience. C'est-à-dire que si je perçois des distinctions en analysant ces images, d'autres vont-ils les percevoir? La meilleure façon de le faire est de faire venir un très grand nombre de gens dans un espace pour leur présenter ces images et voir si, comme moi, ils vont percevoir les contrastes qui existent entre ces images. C'est pourquoi j'ai accueilli avec un très grand plaisir la possibilité de réaliser cette expérience dans l'expérience avec toutes les difficultés que pose une expérience de ce type-là. Ne me bornant pas à une partie du monde ou à une époque, l'expérience nécessitait un très grand nombre d'images différentes. Par exemple, nous avons pu bénéficier du concours du musée du Louvre qui nous a prêté des tableaux. J'étais éperdu de bonheur par le fait de voir de grands tableaux du musée du Louvre côtoyer des fétiches et des masques amazoniens. L'idée était, parmi bien d'autres, de monter qu'il n'y a pas de hiérarchie ou de progression dans les images, mais qu'au fond chaque image ou chaque classe d'image reflètent à sa manière ces contrastes que j'avais mis en évidence dans les textes. C'est pour cela que j'ai souhaité m'entourer d'un très grand nombre d'images de nature très différente en les mettant toutes sur le même plan, ce qui permettrait autre chose aussi. Les anthropologues ont un problème avec l'art qui est une catégorie occidentale. S'il est bien évident que les anthropologues peuvent être très admiratifs des qualités esthétiques d'un objet qu'ils observent sur le terrain et même montrer comment dans une culture des objets sont appréciés de façon différentielle, cette catégorie de l'art est néanmoins est un peu compliquée. Elle l'est d'autant plus qu'après Duchamp il est un peu difficile de qualifier objectivement quelque chose comme un objet d'art. Donc, parler d'images de nature extrêmement différente (buste romain, tableaux de paysages hollandais), considérer et traiter sur un même plan permettait de montrer que l'art n'est pas une catégorie tout à fait pertinente pour analyser les images tel que je souhaitais le faire.

\section{Mme Hélène FULGENCE}

11 Vous présentez votre pensée de manière très claire, mais dans une exposition, le visiteur est seul, vous n'êtes pas là pour lui parler. Vous souvenez-vous de vous être confronté à des enjeux de scénographie, de discours textuel sur un propos qui est limpide lorsque l'on vous écoute, mais qui n'est pas présenté tous les jours dans une exposition temporaire pour des visiteurs qui ont des niveaux de culture et des backgrounds extrêmement diversifiés comme dans tous les musées? Vous souvenezvous de trouvailles, de la scénographie, de textes?

\section{Philippe DESCOLA}

12 Comme tous les anthropologues, je suis d'abord un ethnographe. La première chose que j'ai faite était de voir comment les gens visitaient une exposition, et notamment comment les gens visitaient les expositions de cet espace particulier qu'était la mezzanine.

\section{Mme Hélène FULGENCE}

13 Pour votre information, il s'agit de la galerie ouest où les expositions durent entre 10 et 17 mois. Ce sont donc des expositions plus longues que le rythme habituel qui est de quatre mois.

\section{Philippe DESCOLA}


14 J'ai été me poster, notamment lors de la première exposition Qu'est-ce qu'un corps?, pour voir comment les gens visitaient l'exposition. Je me suis aperçu qu'ils la visitaient complètement à tort et à travers et qu'il fallait donc les contraindre par un dispositif qui n'allait pas les laisser flotter ou errer sans comprendre le propos de l'exposition. J'ai donc imaginé d'avoir une première salle qui présenterait de façon assez didactique, mais pas trop pesante la problématique que je viens d'exposer en l'illustrant par quatre images assez spectaculaires. Ensuite, de présenter les quatre ontologies incarnées dans des images à la suite, mais en ménageant des espaces de superposition de façon à montrer qu'il ne s'agissait pas de façons complètement séparées de voir les choses, mais que des correspondances ou des transitions étaient possibles. C'est là le grand plaisir pour quelqu'un qui, comme moi, était complètement ignorant de ce que c'était que de faire une exposition, c'était de travailler avec les scénographes, avec tout le personnel technique, les socleurs, les éclairagistes, et d'inventer des solutions expérientielles pour tenter de faire passer au public un message qui ne pouvait pas uniquement passer par des objets exposés dans des vitrines. Pour un anthropologue, les images sont des éléments d'une performance. La plupart des images avant l'apparition de l'art sont des objets rituels qui sont insérés dans des cérémonies, dans des pratiques collectives dans lesquelles l'image elle-même, ce qu'elle représente, son iconicité doivent se combiner à de la musique, à des chants, à des énoncés, à des odeurs, à de la douleur parfois dans les rites d'initiation. Tout cela est mis entre parenthèses dans le fait de présenter l'image dans une vitrine. Donc, tout ce qui fait qu'une image agit sur nous, qu'elle nous saisit, qu'elle nous conduit à imaginer des choses, à lui prêter une vie propre, tout cela disparaît. Il y a une énorme déperdition qu'il fallait donc essayer de récupérer. Prenons un exemple. La première salle était consacrée à l'animisme, c'est-àdire l'inverse du naturalisme. Le naturalisme est ce qui s'est mis en place à partir du XVII ${ }^{e}$ siècle en Europe. C'est l'idée que les humains sont complètement singuliers, qu'ils ont une intériorité, le langage, la capacité symbolique et qu'ils différent de toutes les autres entités du monde par cela, mais qu'en revanche, du point de vue de leurs caractéristiques physiques, ils ne se différencient pas véritablement en nature, seulement par des formes d'organisation. L'animisme, c'est l'inverse. C'est l'idée que la plupart des êtres du monde peuvent manifester une intériorité, mais que du point de vue physique chaque classe d'êtres, du fait de ses dispositions particulières, a accès à des mondes singuliers et chacun de ces mondes est un prolongement des capacités et des dispositions de cette classe d'êtres. Pour pouvoir montrer cela à propos de l'animisme, il fallait montrer comment il peut y avoir de l'intentionnalité partout. Une des façons de le faire a été d'inventer un dispositif. La pièce était blanche avec des voiles opaques, mais derrière lesquels il y avait de la lumière. Les vitrines étaient incrustées dans ces parois. Il fallait inventer un système qui fasse varier la lumière de façon aléatoire, mais de manière insensible et donc, susciter un sentiment de bizarrerie chez le visiteur qui voit que son environnement change sans qu'il ne prenne exactement conscience des raisons pour lesquelles il change. Pourquoi? Parce que l'expérience phénoménale de l'animisme est fondée sur le fait que l'on se trouve par exemple face à un animal qui vous regarde au lieu de s'enfuir, à un arbre qui fait un bruit bizarre alors qu'il ne devrait pas faire de bruit. Toutes ces petites bizarreries sont le fondement de l'expérience de l'animisme qui est ensuite systématisée par les ethnologues en théorie. Du point de vue sonore dans l'audioguide, j'avais veillé à ce que l'on mette en fond sonore de façon très discrète des pas qui crissent dans la neige. Pourquoi ? Parce que Carpenter, qui a fait une très belle exposition ici, qui est l'un des 
fondateurs de l'anthropologie du paysage sonore avait mis en évidence il y a longtemps déjà à quel point chez les Inuits en général autour du Pôle Nord, la question sonore joue un rôle important. En particulier lorsqu'il y a du brouillard, on se déplace dans un univers de sons. L'idée du pas qui crisse sur la neige devait pouvoir rendre vivace cette impression. Ce sont de petites trouvailles de ce type-là qui m'ont beaucoup amusée. Autre trouvaille, ce sont ces grands tableaux dans la section sur le totémisme qui sont connus de tout le monde depuis le développement d'un marché de l'art aborigène, ces grands tableaux des sociétés aborigènes d'Australie centrale, un peu pointillistes,et que l'on voit toujours accrochés sur des murs et qui en réalité sont un développement de pictographies qui sont tracées sur le sable. Il m'avait semblé intéressant de les montrer de façon contre-intuitive pour nous, mais de façon plus logique pour les Australiens à plat sur le sol.

\section{Mme Hélène FULGENCE}

15 Je reviens à la question du son que vous évoquiez précédemment à propos des collections permanentes.

Nous voyons comment l'exposition Médias pas si pauvres vous a permis d'expérimenter, d'aller plus loin dans votre recherche, puis de présenter la façon dont les images peuvent être considérées de manière très inhabituelle pour des visiteurs européens dans votre exposition.

Donnons la parole à Lilian Thuram. Au moment où nous nous sommes rencontrés pour la première fois pour travailler ensemble sur votre exposition, vous me dites que les choses se sont déroulées en deux temps pour vous : dans un premier temps, vous avez voulu démonter les mécanismes du racisme et les a priori racistes. Vous allez dans les écoles et vous demandez aux enfants de réfléchir à ce qu'est un homme noir, un homme rouge, un homme jaune et à leurs caractéristiques. Vous posez des questions et l'on vous répond toujours: "Les Noirs sont très sportifs. Les Rouges dansent bien autour du feu. Les Jaunes sont bons en arts martiaux ». Vous posez des questions, vous faites un peu de maïeutique socratique et assez vite vous démontez l'idée qu'il y aurait des races. Ensuite, vous rencontrez à Barcelone Pascal Blanchard qui est l'auteur d'un travail très riche sur la question des exhibitions humaines dans les expositions coloniales que l'on appelait les «zoos humains ». Là, vous comprenez que c'est un moment clé. Il y a bien sûr plusieurs moments historiques qui édifient l'a priori raciste et l'idée que la race blanche dominerait les autres. Celui-là est central. Pascal Blanchard et vous rencontrez Stéphane Martin et vous lui proposez une exposition sur le thème des Zoos humains : nous allons créer une équipe autour de vous, constituée de Pascal Blanchard, historien, et de Nanette Snoep qui est à cette époque Responsable des Collections Histoire du musée du quai Branly - Jacques Chirac. Ce commissariat en triptyque va édifier une exposition, à partir de la question des zoos humains et des exhibitions, puis la dépasser, pour arriver à la question plus globale du préjugé. Le thème de l'exposition a été évidemment très élargi. L'exposition a duré six mois et a accueilli plus de 300000 visiteurs. C'est un succès très important. Pouvez-vous nous dire comment vous êtes passé de cette intention : « Nous allons faire une exposition sur les Zoos humains" à la manière de réfléchir plus globalement sur les préjugés? Et également nous parler de votre travail avec les scénographes, de vos réflexions de l'époque sur la façon de conduire le visiteur dans cette thématique?

\section{Lilian THURAM}


Il est vrai que c'est la rencontre du livre de Pascal Blanchard qui a changé les choses et puis c'est également la rencontre de M. Martin, parce que c'est lui qui m'a demandé de piloter ce projet, de devenir le commissaire général. J'ai essayé de lui expliquer comment je voyais les choses, c'est-à-dire que pour moi l'exposition nous a amenés à nous questionner, non pas seulement sur ce qui s'est passé, mais aussi sur ce qu'il en reste, à la fois dans notre inconscient individuel et dansnos sociétés. Je pense que l'on est quand même plus intelligent lorsque nous sommes plusieurs. Pascal Blanchard et Nanette Snoep étaient plus spécialistes des zoos humains, notamment Pascal. J'essayais toujours de ramener cette histoire-là aux hiérarchies qui existent dans nos sociétés, au fait que nous sommes encore une fois, de façon inconsciente, enfermés dans des groupes. C'est pour cela que nous avons terminé avec le travail de Vincent Elka qui nous questionnait sur les préjugés que nous avons sur les personnes de sexualité, de religion, de genre différents des habitudes dominantes. Parce que pour moi, c'était vraiment cela, c'est-à-dire prendre les visiteurs en leur expliquant qu'il y avait une histoire du racisme. Très souvent, lorsque je vais dans les écoles, les enfants ne comprennent pas que le racisme n'est pas quelque chose de naturel, que c'est une construction politique et économique. Voilà pourquoi nous avons commencé par l'arrivée de Christophe Colomb aux Amériques. Nous avons expliqué dans l'exposition que Christophe Colomb n'avait pas découvert les Amériques. Très souvent lorsque je vais dans les écoles je demande aux enfants qui est Christophe Colomb et ils me disent: "C'est celui qui a découvert l'Amérique ». Je leur dis : «Nous allons faire un petit jeu. Nous sommes dans cette classe. Imaginons que quelqu'un ouvre la porte et dise : «J'ai découvert la classe»». Les enfants me disent: "Cela ne fonctionne pas». Je dis: «Pourquoi? ». "Parce que nous sommes déjà dans la classe. Je leur explique : « Avec Christophe Colomb, c'est exactement la même chose ». Pour moi, cette exposition, c'est un mélange du travail que jefais avec les enfants, du travail que je fais par les écrits et les rencontres avec les étudiants dans les universités, c'est-à-dire essayer d'amener vraiment une réflexion personnelle. Voilà pourquoi nous avons imposé un sens de visite. Il n'était pas possible de visiter l'exposition en allant à gauche ou à droite à partir du sas d'entrée. C'était un ordre chronologique. Cette chronologie nous a vraiment amenés à comprendre que cette affaire des zoos humains est tout de même assez incroyable. D'ailleurs, la grande majorité des gens n'avait pas conscience de cette histoire-là. Je reste persuadé que s'il n'y avait pas eu des images, la plupart auraient dit que ce n'est pas vrai et que cela ne s'est pas passé de la sorte. Nous avons aussi fait le choix, notamment Nanette, de placer dans le parcours des miroirs déformants, parce que là aussi cela vous interpelle. C'est-à-dire qu'à tout moment dans l'exposition il y avait ce regard. Vous tombez devant des miroirs et vous vous questionnez. Je me regarde, que se passe-t-il ? Par moment, il y avait juste une petite photographie. L'on était obligé de s'approcher et de lire. Par cette photographie, on voyait la violence des exhibitions et comment elles ont inscrit le racisme en nous. Pour moi, comment je perçois les autres et comment je me perçois est très important. Il s'agit de savoir s'il y a un rapport entre ce que je vis aujourd'hui et cette exposition-là. Tout au long de l'exposition, c'est ce que nous avons essayé de montrer. Ce qui était assez fort, c'est que nous voyions des gens s'arrêter devant des images et être vraiment bouleversés. Ce qui est aussi très intéressant, c'est que cela libérait les paroles. Très souvent, je venais dans l'exposition pour regarder et discuter avec les gens. J'ai notamment rencontré une famille antillaise qui était extrêmement bouleversée. Ils étaient très contents de pouvoir comprendre que le racisme qui existe aujourd'hui avait une histoire. Je crois 
que l'on ne se rend pas compte qu'aujourd'hui les catégories qui se sont constituées pendant l'histoire deszoos humains existent toujours.

\section{Mme Hélène FULGENCE}

Dans votre travail à tous les trois, avez-vous rencontré des moments de débat? Dans l'exposition, il y avait évidemment la question des zoos humains, mais il y avait aussi toutes sortes d'exhibitions dans des cirques, dans des théâtres, particulièrement au $\mathrm{XIX}^{\mathrm{e}}$ siècle. On autorisait les visiteurs à prendre un ticket pour aller voir d'autres êtres humains. Il y a une ambiguïté très forte dans l'achat de ce genre de ticket et dans ce genre de spectacle. Je me suis toujours demandé si vous aviez débattu pour savoir jusqu'où l'on montre les choses : faut-il sortir de l'ambiguïté et montrer que c'est une démarche condamnable ou au contraire assume-t-on l'ambiguïté de ce type de spectacle et le voyeurisme qui s'y déroule ? Y a-t-il aussi des choses que vous avez décidé de ne pas montrer?

\section{Lilian THURAM}

Bien sûr. De toute façon, il y a eu débat notamment sur le moulage de la Vénus hottentote. Par exemple, Pascal Blanchard voulait absolument le montrer. Nanette et moi n'étions pas très d'accord. Encore une fois, je pense qu'il faut travailler avec intelligence, c'est-à-dire que j'étais là pour prendre la décision finale. Comme nous avons fait un vote, nous avons décidé de ne pas la montrer, parce que connaissant l'histoire de la Vénus, sachant que ce qui restait de son corps avait été rapatrié en Afrique du Sud, je pensais qu'il était plus respectable pour elle de ne pas montrer ce moulage. Il y a eu des débats tout au long de la conception de l'exposition et jusqu'à la fin pour essayer d'être le plus juste possible. Dans tout travail, cela me paraît nécessaire. Montrer l'ambiguïté était extrêmement important pour nous, montrer que le racisme, le sexisme, l'homophobie sont des choses liées à notre culture et que nous intégrons de façon inconsciente. Parfois, on peut le tolérer comme lors de repas de famille. C'est pour cela que l'on ne culpabilisait pas les personnes qui avaient visité à l'époque, parce que c'était un spectacle, mais vous repartiez chez vous en ayant intégré le préjugé de la domination blanche. Tout au long du parcours, pour moi, ce qui était extrêmement important, c'était de montrer le discours de l'époque, replacer les choses dans leur contexte et montrer que même les savants cautionnaient ce regard-là. Cela nous oblige donc à nous questionner sur ce que nous cautionnons aujourd'hui. C'est cela la réalité. Chacun de nous est-il capable de se lever contre certains discours?

\section{Mme Hélène FULGENCE}

Merci, Lilian. Roger, j'ai d'abord fait ta connaissance par ton exposition Cannibales et Vahinés avant de te connaître personnellement. Je voudrais t'interroger, comme je l'ai fait avec les deux autres commissaires, sur le média exposition. Je voudrais partir de Tarzan, parce qu'à un moment donné, l'idée a germé dans notre programmation de travailler sur les représentations de l'altérité dans les pop cultures qui sont des endroits de représentations parfois caricaturaux, mais aussi très profonds. Nous nous sommes lancés dans une exposition. Le choix de cette programmation est lié au fait que nous avions rencontré un collectionneur qui avait des planches originales absolument merveilleuses. L'exposition est un média exigeant, parce qu'il faut avoir des choses fortes à montrer. Tu as accepté d'être le commissaire de cette exposition. À partir d'une exposition qui a l'air pop et qui a l'air de s'adresser au public familial. Comment as-tu travaillé pour faire des choses amusantes, pop et en même temps mettre en jeu des questionnements plus philosophiques? 


\section{Roger BOULAY}

Tarzan parle d'Afrique et moi, j'arrivais du monde océanien. Ce souci de travailler sur l'imagerie, sur les stéréotypes qui sont trimballés par la littérature populaire, par le cinéma, par le disque, etc., depuis la fin du XVIII ${ }^{e}$ siècle ne me vient pas d'un rêve soudain, mais vient d'une expérience. C'est l'expérience que j'ai pu vivre en NouvelleCalédonie avec le monde kanak et en particulier avec la rencontre avec Tjibaou et les gens qui travaillaient avec lui. Les multiples soucis de Jean-Marie Tjibaou, c'était quand il m'a envoyé faire les inventaires dans les musées, une des trois questions était: que dit-on de nous dans ces vitrines? Que raconte-t-on des Kanaks dans ces vitrines? J'ai bien pris soin de garder en moi cette question et j'ai essayé d'y répondre le plus profondément possible et le plus souvent possible, dans des expositions en particulier. Suite à ce questionnement avec Emmanuel Kasarhérou, qui était à l'époque directeur du musée de Nouméa, nous avions monté un petit cycle de trois expositions sur le regard occidental sur les Kanaks; expositions qui se sont faites en préfiguration du Centre Tjibaou. La première de ces expositions questionnait la photographie ethnographique. En ce qui concerne la deuxième, il y avait quelque chose sur l'imagerie, sur la photographie. La troisième s'appelait déjà Cannibales et Vahinés, puisque je l'ai d'abord faite à Nouméa à la demande du Centre Tjibaou avec toujours la question en suspens de Tjibaou: «Que dit-on de nous chez vous?». J'ai pu faire cette exposition dans toute son extension maximum au musée de la Porte Dorée. J'ai pu me régaler en découvrant ce monde des scénographes, des gens qui travaillaient sur ces expositions. Il s'agissait de Philippe Délis, un grand scénographe disparu maintenant, qui, dès cette exposition, a peut-être branché sur le très contemporain zapping généralisé et a mené à faire une exposition qui n'avait pas de sens de lecture. Donc, chacun allait piquer ce qu'il voulait un peu partout. J'étais rendu sensible à toutes ces problématiques du regard dans la littérature populaire, dans le disque, dans le jouet au moment où Stéphane et toi m'avez demandé si j'étais intéressé par le fait de faire une exposition Tarzan. Pour cette exposition, il n'était pas question de faire une histoire de Tarzan ou une sorte de grande revue pour amateurs de bande dessinée de haut niveau des différentes évolutions de la figure de Tarzan dans le dessin, mais de faire en sorte que l'on puisse s'interroger sur la vision de l'Afrique que ce super héros pouvait avoir provoquée dans le public occidental américain et français en l'occurrence. Je me souviens très bien d'une réflexion d'un journaliste américain qui était venu et qui avait dit: «Cette exposition est vraiment un regard gaulois sur le personnage de Tarzan ». $\mathrm{Au}$ cours de cette exposition, j'ai eu la surprise de faire connaissance épistolairement avec le dernier Tarzan existant, Denny Miller, qui avait, tout à l'encontre de son compatriote, envoyé des lettres de remerciements sur le fait que l'on ait pu prendre en compte ce héros américain et en parler en Europe avec apparemment un angle de vue différent lui aussi. La difficulté était de tricoter le discours de l'exposition de manière à ce que chacun y trouve son compte, que chaque public se sente concerné, des enfants aux grands-parents. Je fais cela habituellement en semant dans l'exposition une multitude d'incises dans lesquelles chacun est censé trouver son bonheur et matière à réflexion.

\section{Mme Hélène FULGENCE}

Je ne sais pas si tout le monde a vu l'exposition Tarzan, mais à l'époque nous avions tout de même réalisé une cabane dans le jardin du musée, avec un système d'enregistrement 
où l'on pouvait faire le cri de Tarzan. Beaucoup de visiteurs sont allés s'enregistrer poussant le cri de Tarzan. de vieux Kanaks a visité cette exposition. Ils ont eu comme première réflexion: "Mais c'était aussi grave que cela? », puisqu'il y avait une masse de documents. L'impression qu'ils avaient était qu'il y avait deux ou trois documents et trois ou quatre images qui parlaient mal d'eux. Devant la masse de documents, ils se sont rendu compte de l'importance de ce qui pouvait ressembler à une opération de propagande massive sur eux-mêmes. Comme pour s'excuser que l'on parle de choses aussi terrifiantes que le cannibalisme de leurs ancêtres, ils ont dit: "Tu sais, Roger, il ne faut pas t'inquiéter, c'est du temps où nous étions dans les ténèbres. Cela ne compte plus maintenant. Il faudra parler de nous d'une autre manière ». C'était une petite réaction assez marrante. Il y en a eu d'autres, surtout à Nouméa et ici aussi, puisque cette exposition à la Porte Dorée avait eu une résonance assez importante dans un certain nombre de communautés.

\section{Philippe DESCOLA}

J'ai dit que cette exposition était une expérience dans l'expérience. Les résultats expérimentaux étaient donc les commentaires des visiteurs, parce que j'ai fait visiter à plusieurs reprises l'exposition à des publics très différents, à des classes de collèges et de lycées, à des collègues professeurs au Collègue de France. Donc, il y avait tout l'éventail possible des réactions. Et puis, il y avait surtout ce que disaient les conférenciers qui faisaient visiter l'exposition, ainsi que le livre d'or. Dans le livre d'or, il y a deux commentaires que j'ai trouvés très intéressants. L'un, à l'évidence d'une petite fille, disait: "Quand je serai grande, je serai une animiste ». Il y a matière à écrire un livre là-dessus. Le second, qui était la justification de l'exposition, était : «Cela me permet de voir le reste du musée d'une façon différente ». C'est au fond cela l'idée des expositions temporaires, c'est de servir de contrepoint vis-à-vis des collections permanentes. D'une manière plus générale, c'était pour moi une façon d'avoir accès à un public considérable.

\section{Mme Hélène FULGENCE}

L'exposition a accueilli 275000 visiteurs.

\section{Philippe DESCOLA}

Par rapport à l'auditoire que l'on peut atteindre dans des cours même retransmis sur le web ou dans des écrits, c'est une audience considérable. La réaction générale est que les historiens de l'art étaient très perplexes, car c'était une façon d'aborder les images très différente de celle de l'histoire de l'art, c'est-à-dire avec des généalogies, des ruptures, des mises en contexte culturel, avec les événements politiques et les conditions économiques de l'époque, les transmissions de secrets d'atelier. Au fond, tourner le dos, 
comme le font les anthropologues, à ce type d'historicisation les avait mis très mal à l'aise.

\section{Mme Hélène FULGENCE}

C'est très intéressant que vous rappeliez cela, car j'étais allée rencontrer le directeur du Département des peintures du Louvre pour expliquer de quoi allait traiter votre exposition. Je me souviens très bien qu'à ce moment-là je me disais : "Pourvu qu'il accepte, parce que nous allons utiliser des icônes qui sont dans les salles du Louvre et nous allons les déplacer et leur faire jouer un rôle dans un tout autre discours. Cela ne va-t-il pas agacer?». Finalement, cela n'a pas été le cas.

\section{Philippe DESCOLA}

Vincent Pomarède, qui était le responsable du Département des peintures, a accepté. J'ai été très surpris.

\section{Mme Hélène FULGENCE}

Peut-être parce que le champ de l'anthropologie reste pour les historiens de l'art un champ différent où les choses sont permises parce que l'on est sur un autre territoire.

Lilian, je reviens sur la question de la réception de l'exposition. Elle a eu un succès incroyable. J'ai une anecdote sur la réception de votre exposition : un de mes amis y a emmené ses deux enfants adolescents et il m'a dit que son fils avait été absolument bouleversé et qu'il avait déclaré à son père: "C'est un grand moment de mon éducation ».

\section{Lilian THURAM}

Il est vrai que l'exposition a été une réussite. D'ailleurs, c'était assez risible lorsque nous avons reçu le Globe de cristal au Lido.

\section{Mme Hélène FULGENCE}

Il y avait des remises de prix sous la forme d'un corps de femme dans du cristal dépoli de type dôme. Magnifique. Assez érotique d'ailleurs. Notre directeur général de l'époque s'était trouvé devoir aller chercher ce prix pour l'exposition et pour le musée. C'était un grand moment comique.

\section{Lilian THURAM}

Ce qui est très intéressant, c'est que le public qui a visité cette exposition, qui était très nombreux, n'avait pas l'habitude de venir au musée du quai Branly. Je reste persuadé qu'il y a des discours qu'il faut emmener dans les musées pour attirer de nouveaux visiteurs. Beaucoup de personnes m'ont remercié à la sortie, parce que cela les apaisait. Je suis dernièrement venu visiter l'exposition sur M. Chirac. Une dame, qui travaille depuis très longtemps au musée, est venue me voir en me disant: "Depuis cette exposition, le discours du musée a évolué ». C'est un très beau cadeau. Cela veut dire que cette exposition a peut-être permis de dédramatiser certaines choses.

\section{Mme Hélène FULGENCE}

37 J'ai envie de vous poser encore trois questions. Quel est votre plus mauvais souvenir d'exposition en tant que visiteur?

\section{Philippe DESCOLA}

En général, l'on cherche à les effacer.

\section{Roger BOULAY}


J'ai un exemple très récent, puisque c'est à propos de l'exposition Sepik. J'ai eu l'occasion de visiter cette exposition dans un autre endroit et j'ai été extrêmement déçu. Je me suis dit : «J'espère qu'à Paris ce ne sera pas comme cela ». La personne qui était avec moi, en guise de critique absolue et définitive de l'exposition, m'a dit : «Il manque les prix d'affichés sur les objets ». Comme quoi les options muséographiques et scénographiques sont vraiment fondamentales. L'intérêt de ce média est de travailler avec des scénographes et des gens qui vont peaufiner votre discours, l'amender, vous dire : «Cela est incompréhensible. Cela est faisable, cela n'est pas faisable ». C'est ce qui est passionnant.

\section{Mme Hélène FULGENCE}

Quel est votre meilleur souvenir d'exposition?

\section{Philippe DESCOLA}

41

Il est très difficile de répondre à l'une ou l'autre des questions. Je suis très bon public et je trouve toujours que les choses sont géniales. Souvent, quand je lis, je trouve que c'est très intéressant et que c'est beaucoup mieux que ce que je fais. Je ne vois pas pourquoi je me casse la tête à écrire des choses alors qu'il y en a d'autres qui font des choses beaucoup mieux que moi. Au fond, il n'y a pas d'optimum pour cela. Je me souviens de la Biennale de Venise il y a six ans, j'avais été frappé à la fois par une très mauvaise scénographie - il n'y avait rien. C'était une sorte de hangar - et en même temps par l'extraordinaire inventivité de ce qui était présenté. À l'évidence, la scénographie ne jouait pas tellement un rôle si les objets étaient puissants ou les assemblages. Et puis, à côté de cela une scénographie très élaborée, mais qui tournait à vide. Au fond, je crois qu'il n'y a pas de solution miracle.

\section{Mme Hélène FULGENCE}

\section{Lilian THURAM}

Une exposition qui m'a plu était Présence africaine. Cela a été un choc. J'ai trouvé cela magnifique.

\section{Roger BOULAY}

J'ai beaucoup aimé Jazz. Et puis, par rapport au travail que nous faisons et que je fais sur cette imagerie, je dois rendre hommage à des musées de province qui font ou qui initient ce travail. Ma première rencontre avec ce type d'idée, c'est au musée de Brême dans les années 1980. Ce musée avait une énorme vitrine d'objets fabriqués pour le tourisme en particulier et d'objets océaniens. C'est encore une fois la masse de ces productions qui m'a fait penser qu'il y avait quelque chose à faire là, sachant aussi que j'ai entendu tellement de choses dans ma période Mao autour des poteaux de torture exposés dans la grande salle dite des totems. Il y avait un maximum de lieux communs. Donc, un hommage à ce musée. Et puis, des musées comme celui d'Angoulême qui ont une vitrine permanente consacrée à ces stéréotypes, à cette imagerie autour d'objets.

Pause méridienne.

Les actes de colloques du musée du quai Branly Jacques Chirac, 8 | 2017 


\section{AUTEURS}

HÉLĖNE FULGENCE

Directrice du développement culturel, musée du quai Branly-Jacques Chirac

\section{PHILIPPE DESCOLA}

Anthropologue, professeur au Collège de France, commissaire de l'exposition « La Fabrique des images »

\section{LILIAN THURAM}

Président de la Fondation Education contre le racisme, commissaire général de l'exposition «Exhibitions, l'invention du sauvage »

ROGER BOULAY

Ethnologue, commissaire des expositions « L'aristocrate et ses cannibales », « Tarzan » et « Kanak, l'art est une parole». 